ArtefaCToS. Revista de estudios de la ciencia y la tecnología

eISSN: $1989-3612$

Vol. 7, No. 2 (2018), 2a Época, 35-57

DOI: http://dx.doi.org/10.14201/art2018723557

\title{
La justificación de la abducción en el contexto del debate sobre el realismo científico: una aproximación argumentativa
}

\author{
The Justification of Abduction in the Context of the Debate \\ over Scientific Realism: an Argumentative Approach
}

\section{Paula OLMOS GÓMEZ}

Universidad Autónoma de Madrid

paula.olmos@uam.es

Recibido: 18/07/2018. Revisado: 09/09/2018. Aceptado: 09/09/2018

\section{Resumen}

Las nuevas perspectivas sobre la naturaleza de las prácticas justificativas que ha traído el desarrollo de una teoría de la argumentación ni formal ni deductivista nos ofrecen la oportunidad de replantearnos algunas cuestiones centrales en filosofía de la ciencia, revisando nuestro análisis de los modos de dar, pedir y recibir críticamente razones propios de la actividad científica. Las nociones y distinciones propias de dicho marco nos permiten clarificar la estructura racional del discurso probatorio de los agentes científicos que incluye actos argumentativos y explicativos intrínsecamente interrelacionados, lo que determina la centralidad de un esquema argumentativo meta-explicativo como es el de la abducción. Pero hay un debate filosófico que se sitúa más allá de la identificación y comprensión de dichas estructuras racionales y más allá de su evaluación por parte de los agentes participantes en la discusión científica. Tal sería el debate entre filósofos sobre la justificación epistémica de la abducción que, a su vez, se relaciona con las diversas posturas en torno a las posibilidades de justificación del realismo científico. En el artículo se revisan distintas posturas en torno a dicha justificación, exponiendo su sentido argumentativo y el modo en el que condicionan la comprensión de los argumentos abductivos particulares.

Palabras clave: abducción; argumentación; comprensión; explicación; garantía; inferencia de la mejor explicación; justificación; meta-argumentación; modelo de Toulmin; realismo científico. 


\begin{abstract}
The new perspectives on the nature of justifying practices brought by the development of a non-formal and non-deductivistic argumentation theory offer us the opportunity to revisit some central issues for the Philosophy of Science and revise our analysis of the typical ways to give, ask for and critically receive reasons in the Sciences. The use of the notions and distinctions developed by this theoretical framework help us clarify the rational structure of the scientific agents' evidentiary discourse, which includes closely interrelated both argumentative and explanatory acts. This determines the centrality of a meta-explanatory argumentative scheme such is abduction. However, there is a philosophical debate that goes beyond the identification and comprehension of these rational structures and even beyond their assessment conducted by the participants in scientific discussions. This is the debate among philosophers regarding the epistemic justification of abduction, closely related to the different stances regarding the justifying possibilities of scientific realism. This paper revises different viewpoints on the justification of abduction, exposing their argumentative import and the way they govern the comprehension of particular abductive arguments.
\end{abstract}

Keywords: abduction; argumentation; comprehension; explanation; inference to the best explanation; justification; meta-argumentation; scientific realism; Toulmin model; warrant.

\title{
1. Introducción. Las prácticas científicas de justificación racional
}

Sin ánimo alguno de recuperar la exclusiva relevancia del llamado "contexto de justificación" en los estudios filosóficos sobre la ciencia, lo cierto es que la apertura de nuevas perspectivas sobre la naturaleza de las prácticas justificativas que supone el desarrollo de la teoría de la argumentación -que trataría de caracterizar y analizar diversas modalidades de los actos comunicativos de dar razones bajo un marco ni formal ni deductivista- nos ofrece la oportunidad de replantearnos algunas de las cuestiones que llevaron al Positivismo Lógico a algún que otro callejón sin salida.

La constatación del propósito justificativo de una parte importante (si no central) de las prácticas científicas sería, en todo caso, el punto de partida para mostrar la pertinencia de un enfoque argumentativo. Esta idea está muy presente en un reciente trabajo de John Woods (2016), precisamente centrado en la lógica de la abducción, en el que insiste en el carácter forense de la ciencia, utilizando una terminología jurídica que más tarde volveremos a reseñar y que ya estaba presente en la concepción original de Stephen Toulmin, para quien la lógica en su versión argumentativa debía desarrollarse como "jurisprudencia generalizada" (Toulmin, 2003 [1958], 7). De acuerdo con Woods: 
Tal como sucede con el derecho, la ciencia es en gran medida una profesión dedicada a la justificación de sus conclusiones [a case-making profession] -i.e., una profesión forense- y lo es en tanto que recompensa el mostrar que se ha alcanzado cierto conocimiento por encima del mero alcanzarlo [...] La publicación es el medio por el que se realiza la defensa del caso o conclusión y defender un caso es una tarea más exigente que simplemente saber. A los editores de revistas académicas no les importa un pimiento lo que alguien sepa. Pero quizá escuchen y tengan en cuenta lo que alguien pueda mostrar que sabe. (Woods, 2016, 143-144, mi traducción)

El interés por una mirada renovada y desprejuiciada sobre las prácticas científicas de justificación racional, basada en su comprensión como prácticas argumentativas, respondería, por otro lado, al desafío lanzado en su día por Dudley Shapere, que Alberto Cordero reseñaría, de manera muy significativa para nuestros propósitos, en un artículo de 1991, relacionando el enfoque de Shapere con el de Stephen Toulmin y calificando a ambos de defensores de un "antifundacionismo racionalista":

Dudley Shapere, en particular, es el autor de una combinación particularmente robusta de antifundacionismo y racionalismo en la filosofía contemporánea. [...] Shapere sostiene que la epistemología tradicional está en quiebra, no porque la ciencia no haya podido generar un conocimiento basado en la razón, sino porque la filosofía no ha podido comprender acerca de qué tratan el razonamiento y el conocimiento. (Cordero, 1997 [1991]: 209) ${ }^{1}$

En dicho artículo, Cordero utiliza estas directrices para criticar algunas versiones del naturalismo (de W. v. O. Quine a R. Giere, pasando por D. Campbell) que, a su entender, estarían empeñadas en tratar de reducir las prácticas epistémicas de dar razones a la mera puesta en funcionamiento de mecanismos cognitivos indisponibles. Cordero aboga, en cambio, por un naturalismo no reduccionista, militantemente antifundacionista, pero racionalista, en el sentido preciso de atender a las prácticas científicas de justificación racional (prácticas, por otro lado, colectivas de dar razones) que cubrirían la brecha entre la cognición humana explicada en términos naturalistas y el conocimiento social y sistemáticamente sancionado propio de las ciencias empíricas.

Conforme a su convicción naturalista, Cordero, siguiendo en esto a Shapere, estaría dispuesto a delegar en la propia discusión de los científicos y en su reconocimiento consciente de los parámetros de su actividad, la decisión

\footnotetext{
${ }^{1}$ Cito el artículo de Cordero por su traducción al español, a cargo de J.M. Martínez, realizada para su inclusión en el volumen compilado por Sergio Martínez y León Olivé, Epistemología evolucionista (1997).
} 
sobre la corrección y la racionalidad de sus planteamientos particulares, otorgando a la filosofía la tarea de comprender todo el proceso y reseñar el modo en el que, de manera histórica y pragmáticamente situada, se constituyen los usos normativos de la noción científica de razón:

Precisamente porque la racionalidad científica implica una interacción de toma y daca entre el pensamiento y el aprendizaje, porque tiene ese peculiar carácter histórico, es que el discurso acerca de ella no tiene que ser acerca de algo que trasciende la ciencia en su contexto real histórico, teórico y pragmático. Así que, según este enfoque, no podemos entender la racionalidad científica sin ser primero conscientes del pasado y presente racionales de la ciencia. Una vez hecho eso, podemos tratar de entender el uso del concepto contemporáneo de "una razón", atendiendo a la manera en que los planteamientos, los problemas, las posibilidades, los métodos, los fines, etc., se consideran exitosos y libres de dudas, y cómo tales ideas llegan a juzgarse pertinentes para problemas específicos. (Cordero, 1997 [1991], 210-211)

En trabajos posteriores, Alberto Cordero ha seguido insistiendo en el carácter central del antifundacionismo y el falibilismo como rasgos nucleares (más allá de las diferencias) de los enfoques naturalistas en filosofía de la ciencia (Cordero y Galparsoro, 2013). Así, en la introducción al citado volumen colectivo, a cargo de los editores, se defiende explícitamente un concepto naturalista de justificación de carácter explícitamente antifundacionista y falibilista:

La justificación naturalista como tal se apoya en consideraciones como la coherencia, la adecuación con los datos y el éxito de predicciones arriesgadas. Sin embargo, incluso una propuesta exitosa en términos científicos resultaría, en términos de una noción de éxito no naturalista, filosóficamente pobre y desencaminada desde el inicio. El no naturalista se pregunta ¿Qué es lo que justifica el propio método científico al que apelan los naturalistas? Creo que la respuesta de Ernest Nagel a este tipo de críticas sigue siendo rotunda. En los albores del naturalismo contemporáneo, Nagel sostenía que tales objeciones solo preocupan a aquellos que "se niegan a otorgar la dignidad de conocimiento genuino a cuanto no está demostrado a partir de premisas prístinas y autoevidentes" (1956, p. 15). (Cordero y Galparsoro, 2013, 9, mi traducción)

Esto nos permite encuadrar las prácticas científicas de justificación racional como prácticas distintivamente argumentativas, en las que tanto los puntos de partida como las conclusiones se consideran $-\mathrm{y}$ ello por parte de la 
propia comunidad en la que se discuten- por un lado, solo provisionalmente establecidos pero, por otro, razonablemente justificados en el sentido de no suscitar (al menos demasiadas) dudas en el momento en el que se aceptan, situándose, en terminología reconociblemente jurídica, "más allá de la duda razonable" (Cordero, 1997 [1991], 196). Lo cual supone, por supuesto, una caracterización de la justificación en la ciencia pragmáticamente situada y relativa tanto a los medios epistémicos disponibles como a los fines de la investigación y a las condiciones reales de la discusión entre científicos.

Seguramente, no sea este el tipo de concepto de justificación con el que soñaban algunos filósofos, pero es el que razonablemente podemos extraer de la adopción de un naturalismo atento a las prácticas científicas de dar, pedir y evaluar críticamente razones para la defensa de las pretensiones epistémicas de las ciencias.

\section{Argumentación, explicación y abducción}

Ahora bien, parte de la complicación del estudio de las prácticas científicas de dar razones proviene de una característica propia de las ciencias empíricas cual sería su pretensión explicativa ${ }^{2}$. Si bien el objetivo más conspicuo de los científicos - restringiéndonos ahora a sus actividades forenses, en el sentido antes apuntado- sería proponer y justificar teorías, en principio descriptivas, de los mecanismos que subyacen al comportamiento de los componentes del mundo; se entiende que dichas teorías tendrían como propósito último permitirnos explicar, dar cuenta de y hacer comprensibles tales comportamientos. Pero explicar es también una actividad que comporta dar razones.

De hecho, en el marco de la teoría de la argumentación, tanto las prácticas de justificación como las (complejamente cercanas) de explicación se conciben fundamentalmente como actos comunicativos (principal aunque no exclusivamente discursivos o lingüísticos) de dar razones para una determinada pretensión de contenido teórico, evaluativo o práctico. La diferencia estriba en que mientras:

a) un acto de argumentar sería un acto comunicativo en el que se ofrecen razones para justificar una tesis o pretensión que se pone en entredicho, buscando (generalmente) la convicción o persuasión del interlocutor sobre su corrección;

b) un acto de explicar sería un acto comunicativo en el que se ofrecen razones que pretenden hacer comprensible un contenido que se $d a$

\footnotetext{
${ }^{2}$ Una pretensión, por cierto, también enfatizada y defendida por S. Toulmin, en este caso en un libro más afín a las discusiones centrales en filosofía de la ciencia como sería Foresight and Understanding (1961).
} 
por mutuamente aceptado (el explanandum), pero que o bien resulta sorprendente o inesperado, o bien se considera corriente pero de trayectoria causal desconocida, buscando la comprensión del interlocutor sobre su origen, su relación con otros fenómenos o su congruencia, con idea de que supere la sensación de sorpresa o desconcierto ${ }^{3}$.

Los argumentos y las explicaciones serían los productos discursivos (objetivables para el analista) de tales actos y que constarían de ciertas partes identificables (razones/conclusión; explanans/explanandum) que se presentan en los mismos en tanto que vinculadas entre sí por determinadas relaciones que encarnarían y materializarían su potencial justificativo o explicativo.

De acuerdo con esta caracterización, los actos (y los productos) de explicar y argumentar serían estructuralmente similares - en ambos casos se ofrecerían razones que lo serían para un determinado contenido- y pragmáticamente diversos - el estatus de dicho contenido y los fines de la acción serían distintos en ambas actividades (cf. Marraud, 2013, 24-26). Esta semejanza estructural nos permite construir un modelo de explicación calcado sobre el modelo de argumento propuesto por Stephen Toulmin (2003 [1958]; cf. Bermejo Luque, 2016, 408-410). En el modelo de Toulmin para un argumento sencillo o atómico, la razón que respalda una conclusión (y que se identifica con los datos relativos a un caso particular) se presenta como tal en cuanto que vinculada a dicha conclusión por una relación expresable como una regla de inferencia que actúa como garantía del argumento. ${ }^{4}$

Conviene recalcar que, en el modelo de Toulmin, para que algo se presente como razón para una conclusión no tiene por qué implicarla, solo sustentarla o apoyarla. Del mismo modo, la garantía no tiene por qué expresar una relación lógicamente formalizable, como una condición suficiente, necesaria o de equivalencia. De hecho, lo más normal es que no lo haga sino que exprese una generalización del tipo de vínculo que pone en relación los datos con la conclusión. Utilizaremos el siguiente diagrama tipo (basado en Toulmin y desarrollado por H. Marraud, 2017) para representar un argumento:

Garantía:

\begin{tabular}{|c|}
\hline Datos/Razón \\
\hline Por tanto \\
\hline Conclusión/Pretensión \\
\hline
\end{tabular}

\footnotetext{
${ }^{3}$ La distinción entre argumentación y explicación también se articula en torno a la distinción entre tipos de razones justificativas y explicativas (Álvarez, 2010, 2016).

${ }^{4}$ El modelo de Toulmin identifica otras partes de los argumentos atómicos pero "Datos", "Conclusión" y "Garantía" son las partes básicas que nos permiten identificar y empezar a comprender su estructura y pretensiones justificativas.
} 
Conforme a esta misma idea, en el modelo toulminiano de explicación que ya he propuesto en otros trabajos (Olmos, en prensa), el carácter explicativo de las razones ofrecidas en el explanans se sustenta en algún tipo de idea de nivel más general o principio que expresa en qué sentido el explanans explica el explanandum y que puede hacer alusión a alguna idea concreta de qué cuenta como explicativo en el contexto en el que esta se ofrece. Dicho principio actuaría como garantía explicativa y expresaría el modo particular en el que el explanans se consideraría tal para el explanandum, es decir, respondería a la pregunta ¿en qué sentido se considera que el explanans hace más comprensible el explanandum? A la que se contestaría de acuerdo a distintas posibilidades exploradas, en el caso de la ciencia, tanto por los propios científicos como por los filósofos, que habrían ido acuñando para ello conceptos como los de vera causa, mecanismo, unificación teórica, etc. El diagrama correspondiente para representar una explicación sería:

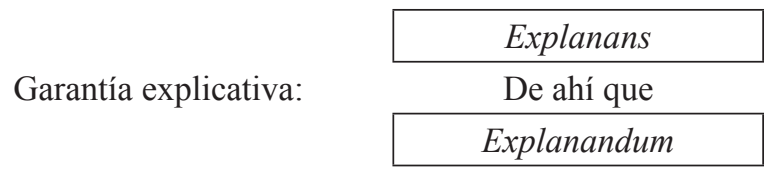

Como era de esperar, tampoco en este caso se requiere que la relación entre explanans y explanandum sea de implicación lógico-formal sino, más bien, sustantiva y sustantivada en la propia garantía explicativa.

Y ahora daremos un paso más que resulta fundamental para comprender, en concreto, las prácticas de justificación en las ciencias empíricas. Pues es obvio que la distinción pragmática entre argumentación y explicación que aquí estamos enfatizando es particularmente crucial para la comprensión de un tipo de argumento peculiar como es el argumento abductivo que, de hecho, las combina, aunque con funciones diversas.

La abducción ha interesado desde hace años a diversos filósofos de la ciencia que la han llegado a considerar como la forma lógica del descubrimiento científico. Para los teóricos de la argumentación, que se centran en los actos comunicativos de justificación y no en los procesos de razonamiento individual, la abducción es un tipo particular de argumento analizable como un esquema argumentativo. ${ }^{5}$ La peculiaridad de este tipo de argumento es que su garantía alude a la posibilidad de construir, con los elementos del mismo, una explicación, de modo que puede representarse del siguiente modo:

\footnotetext{
${ }^{5}$ Para la teoría de los esquemas argumentativos, ver Walton, Reed \& Macagno (2008) y, en español, Marraud (2013, 177-233).
} 
De acuerdo con este esquema, un argumento abductivo es un argumento en el que una conclusión (que en principio consideraremos teórica o fáctica), que típicamente mencionará entidades, propiedades y procesos bien inobservables, o bien meramente no observados, o, sencillamente, no considerados hasta el momento en relación con los datos, se justifica por constituir o contener una explicación concebible para esos mismos datos (empíricos) que se dan por mutuamente aceptados en el contexto en el que dicho argumento se ofrece. Se trata, pues, de un tipo de argumento que sostiene y trata de justificar, al menos en un sentido mínimo y en base a su capacidad explicativa, una hipótesis.

Como vemos, en una abducción, las componentes básicas del argumento se concretan del siguiente modo:

i. La Conclusión/Tesis es una hipótesis explicativa que se expresa, en principio, como un enunciado fáctico, aunque, dependiendo del contexto (y esto es algo que sucede muy especialmente en la ciencia) pueda reinterpretarse como como una pretensión práctica: "deberíamos explorar (poner a prueba) la hipótesis H", "Merece la pena investigar la hipótesis H”.

ii. Los Datos/Razones aducidos son los propios datos observables, empíricos o, en todo caso, dados por aceptados y compartidos en el contexto argumentativo. Sin embargo, su aceptación no zanja las necesidades epistémicas de los participantes, quienes consideran que se trata de datos "sorprendentes" o, en todo caso, que deben ser explicados, i.e. que se constituyen en explanandum. En todo caso, tanto el carácter compartido de los datos como su consideración de explananda pueden ser subsiguientemente discutidos por los interlocutores o receptores de un argumento abductivo, aunque se consideren condiciones básicas de identificación y comprensión del mismo.

iii. La Garantía, lo que hace que los datos sean una razón justificativa de la conclusión o hipótesis, es que la hipótesis podría utilizarse para explicarlos, en un acto explicativo de dar razones que es distinto de aquel en el que se presenta el argumento.

Por ello he defendido en varios trabajos (Olmos, en prensa) el carácter meta-explicativo de la abducción. Si queremos exponer, de manera clara, dicho carácter meta-explicativo, conviene utilizar un diagrama ampliado en el que explicación a la que alude la garantía del argumento abductivo se despliegue 
con sus partes integrantes, lo que nos puede ayudar a localizar posibles elementos en los que se centre la discusión posterior evaluativa ante la presentación de una justificación de este tipo. El diagrama ampliado o desplegado del argumento abductivo tendría la siguiente forma:

\begin{tabular}{l|c|c|}
\multicolumn{1}{l}{} & \multicolumn{1}{c|}{ Razón(es): Datos (empíricos) } \\
\cline { 2 - 2 } $\begin{array}{l}\text { En virtud de garantía } \\
\text { explicativa X: }\end{array}$ & Teoría (hipótesis) & \\
\cline { 2 - 2 } & De ahí que & \\
& Datos & \\
& Conclusión: teoría, hipótesis \\
\hline
\end{tabular}

Un argumento simple abductivo estaría completamente descrito de este modo y sería un tipo característico de argumento con un tipo de garantía característica. Sin embargo, como cualquier otro tipo de argumento, se presentará en una situación en la que sus pretensiones justificativas quedarán sujetas a discusión, una discusión de carácter evaluativo en la que típicamente se presentarán contraargumentos que incidirán en una u otra de sus partes (objeciones, recusaciones o refutaciones, cf. Marraud, 2013, 64 ss.) y en la que pueden solicitarse otro tipo de razones (no necesariamente de tipo abductivo) para mejorar el estatus epistémico de la conclusión y tratar de establecerla "más allá de la duda razonable", si es que ese es y solo en la medida en la que sea nuestro objetivo (e.g. en un contexto científico).

Es habitual y propio de este tipo de argumentos, por otro lado (aunque no necesariamente constitutivo de su definición), que, si el contexto lo permite, la justificación de la hipótesis acabe siendo en algún momento comparativa y relativa a la propuesta de otras hipótesis alternativas, lo que nos obliga a establecerla no solo como una hipótesis adecuada sino además como la mejor hipótesis explicativa disponible. Ello comporta el inicio de un proceso argumentativo y justificativo complejo y, en mi opinión (Olmos, en prensa), no sujeto a una única secuencia posible, que supone la combinación de argumentos y contraargumentos en una interacción, generalmente colectiva y dialéctica, que sería la versión argumentativa de lo que ha venido en llamarse "inferencia de la mejor explicación" (Harman 1965, Lipton 1991, Iranzo 2007). ${ }^{6}$

Centrándonos ahora en las prácticas específicamente científicas de justificación racional, como decíamos anteriormente, es fácil ver que para su caracterización necesitamos entender tanto las estructuras argumentativas o justificativas, como las propiamente explicativas y también el modelo de argumentación que las combina y que sería el de la abducción. Si bien se ha

\footnotetext{
${ }^{6}$ Denominación que por muchas razones expuestas ya en varios trabajos (ver Olmos, en prensa) no considero del todo adecuada.
} 
considerado, en ocasiones, que el discurso científico sería pretendidamente explicativo en términos globales, en el sentido de tratar básicamente sobre explicaciones, conviene distinguir entre:

1. Actividades cientificas (discursivas o no) dirigidas a la búsqueda de explicaciones.

2. Actos discursivos de tipo explicativo, i.e. actos de dar explicaciones: algo que, generalmente, se daría en un contexto didáctico como resultado de la aplicación de teorías científicas establecidas o vigentes.

3. Actos discursivos de discusión/justificación de explicaciones, i.e. actos argumentativos en torno a la justificación de hipótesis explicativas, ya sea en un sentido de propuesta inicial (generalmente asociada a un esquema abductivo) o dentro de un complejo proceso comparativo.

Si pretendemos con los segundos construir y presentar explicaciones cientificas que respondan a la estructura tipo:

Leyes, teorías, regularidades, reconstrucciones,

etc.

De ahí que

Evidencia empírica/experimental, fenómenos/ datos compartidos, resultados constatables

será porque, previamente, los científicos han sido capaces que presentar argumentos a favor de sus conclusiones teóricas y justificarlas por medio de estructuras argumentativas del tipo:

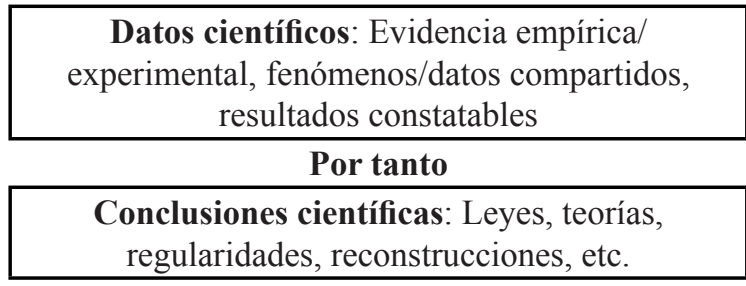

\section{La evaluación de argumentos abductivos}

La metodología de los esquemas argumentativos que mencionábamos anteriormente completa la descripción identificativa de los tipos de argumento, de la que nos hemos ocupado hasta ahora, con una batería de preguntas críticas (critical questions) que permiten su evaluación, es decir, la calificación de 
su desempeño como mejor o peor argumento (más fuerte o más débil) perteneciente a una determinada categoría, sin que un juicio de debilidad haga, en principio, que el argumento deje de serlo, ni le impida seguir perteneciendo a la categoría en cuestión.

Aunque hay varias maneras de entender el papel de las preguntas críticas, aquí las tomaremos como indicaciones (recogidas, analizadas y ordenadas por los teóricos) de las posibles líneas de crítica y contra-argumentación que suscitan las propuestas de argumentos. En el caso específico de los argumentos abductivos, Marraud $(2017,5)$, por ejemplo, ofrece el siguiente listado de preguntas críticas pertinentes:

PC1. ¿Qué grado de certeza debe exigirse a las inferencias en la situación argumentativa dada?

PC2. ¿Sería preferible proseguir el diálogo sin extraer una conclusión en este momento?

PC3. ¿Son fiables los datos aportados?

PC4. ¿Se han tenido en cuenta todos los datos disponibles?

PC5. ¿En qué medida ha sido la búsqueda de datos completa y exhaustiva?

PC6. ¿La hipótesis propuesta es razonablemente sencilla y explicativa?

PC7. ¿Qué otras explicaciones de los datos pueden concebirse?

PC8. ¿Se han considerado todas las explicaciones disponibles?

PC9. ¿Es la explicación propuesta mejor que las explicaciones alternativas?

PC10. ¿Hay razones para pensar que, pese a todo, la hipótesis es falsa?7

Esta batería de preguntas pretende guardar un cierto orden. Las dos primeras (PC1-PC2) son de tipo dialéctico-procedimental y definen condiciones propias del tipo de situación y práctica argumentativas en las que se ofrece el argumento abductivo, i.e. su grado de exigencia de rigor y la existencia o no de la obligación de llegar a una conclusión en un tiempo determinado. Piénsese en las imposiciones temporales de un procedimiento judicial, por ejemplo; una exigencia que, en principio, sería más relajada, aunque no inexistente, en el caso de la actividad científica.

PC3 a PC5 presentan posibles modos de "objeción" sobre los datos iniciales, ya que, aunque las condiciones propias del planteamiento de un argumento abductivo incluyen el presupuesto (por parte del proponente) de que se está

\footnotetext{
${ }^{7}$ Otros autores proponen listados algo diversos y no parece que se trate de listados que puedan "cerrarse" de manera definitiva. 
partiendo de datos empíricos "compartidos" y "constatables", siempre puede suceder que, en el curso de la discusión, surjan objeciones a los mismos, como ya comentábamos anteriormente.

La pregunta PC6 incide sobre la calidad del vínculo propuesto (es decir, mostraría la vía de una posible "recusación" o undercutting defeater en terminología de Pollock, 1987), y su respuesta indicaría la medida en la que asentimos o disentimos sobre la idea de que la hipótesis sea efectivamente una "explicación viable" de los datos. Dependiendo de la situación argumentativa, la pregunta podría sustanciarse en requerimientos más específicos, que exijan la apelación a algún modo o principio de explicación concreto (el principio mencionado en la "garantía explicativa" de la "garantía argumentativa" de la abducción) que resulte aceptable o incluso institucionalmente aceptado en el campo en cuestión (e.g. en el caso de la ciencia, la invocación a mecanismos, verae causae, etc.).

La pregunta PC10 apunta la posibilidad de una clásica refutación (rebutting defeater), i.e. la búsqueda de argumentos alternativos que falsifiquen la conclusión o hipótesis explicativa. De recibir PC10 una respuesta positiva, una propuesta de argumento abductivo, con su carga justificativa, se vería enfrentada a otro argumento (que podría ser de cualquier tipo, i.e. aportar cualquier tipo de razón o evidencia) cuya conclusión resultaría ser explícitamente contradictoria. Si dicho contraargumento resultase vencedor en tal enfrentamiento, la abducción quedaría refutada.

En cuanto a las preguntas PC7-PC9, nos encontramos en ellas con la complicación de que invocan la posible existencia o la constatación de hipótesis explicativas alternativas. Empezar a contestar a dichas preguntas nos lleva por una vía comparativa de evaluación de la propuesta inicial abductiva que iniciaría característicos procesos de ponderación que he descrito en otros trabajos (Olmos, en prensa).

Como vemos, el enfoque argumentativo permite arrojar más luz sobre la distinción entre la abducción, como modo de argumentación relativamente unitario y que responde a un tipo de esquema concreto, de carácter meta-explicativo, y los procesos, más variados, de justificación de la mejor hipótesis explicativa, generalmente correspondientes a "estructuras argumentativas" complejas. Conforme a tal marco, ni se deniega a la abducción simple su componente justificativa (puesto que pone en marcha un "tipo de razón" específico), ni se asimilan los procesos de ponderación de hipótesis a un único esquema, lo que resulta poco operativo a la vista de la diversidad de prácticas argumentativas que encontramos incluso en los terrenos privilegiados de la ciencia. 


\section{Debates filosóficos en torno a la justificación de la abducción}

En todo caso, hasta aquí, hemos partido del presupuesto de que los argumentos abductivos son evaluables, es decir, son susceptibles, una vez identificados o interpretados como tales, en un contexto discursivo de tipo probatorio o forense, de ser sometidos a un proceso, a su vez argumentativo, de cuestionamiento - mediante la puesta en marcha de preguntas críticas, contra-argumentos y ponderaciones que comentábamos en la sección anterior-; un proceso de cuestionamiento del que pueden salir más o menos indemnes, reforzados, debilitados o rechazados, lo que, a su vez determina la suerte de sus conclusiones para ser o no establecidas "más allá de la duda razonable".

Así, las conclusiones de los argumentos abductivos propuestos en un contexto de indagación científica, como hipótesis explicativas, pueden ser, finalmente, aceptadas - por considerarse exitosas y "libres de dudas", conforme a la caracterización de Cordero (1997 [1991], 211- o rechazadas, pero puede también que se suspenda temporalmente en juicio sobre ellas, pueden permanecer en el debate científico como conjeturas plausibles, como hipótesis dignas de someterse a prueba, etc. Las prácticas científicas reales de justificación racional nos pueden proporcionar ejemplos de todo ello.

Hay, sin embargo, un debate filosófico que se sitúa más allá de la identificación y comprensión de las estructuras racionales del discurso probatorio de la ciencia e, incluso, más allá de su evaluación por parte de los agentes participantes en la discusión científica. Tal sería el debate entre filósofos sobre la propia justificación epistémica de la abducción que, a su vez, se relaciona con las diversas posturas en torno a las posibilidades de justificación del realismo científico. En dichos debates filosóficos, no se trata tanto de ver cuáles son los modos adecuados de considerar una propuesta abductiva particular como más o menos exitosa y libre de dudas, sino de poner en cuestión el propio fundamento lógico de la abducción. No se trata tanto de comprender cómo funciona la evaluación de abducciones particulares, cuanto de explorar la justificación, con vistas a su aceptación o rechazo, de la abducción misma (en la línea tradicional de problemas paralelos como el de la "justificación de la inducción").

A pesar de las mencionadas advertencias de Cordero y Galparsoro (2013, 9) sobre el carácter dudosamente naturalista de tales preocupaciones, que parecen actuar como motivación de una empresa en busca de un fundamento epistémico último y firme, lo cierto es que un análisis de las distintas posturas filosóficas en torno a las posibilidad o imposibilidad de justificación de la abducción, realizado con las herramientas que nos proporciona la propis teoría de la argumentación, puede ayudarnos a exponer, precisamente, su sentido argumentativo, es decir, el modo en el que condicionan la comprensión y la evaluación de los argumentos abductivos particulares. 
Dentro de este marco teórico, la discusión en torno a la "justificación de la abducción" se plantea como la problematización de la aceptabilidad de las garantías inferenciales (en el sentido de Toulmin) que permiten, de hecho, presentar argumentos abductivos. La mejor manera de entender las distintas posturas en torno a dicha aceptabilidad (muchas de ellas asociadas, precisamente, a la defensa o ataque del realismo científico) es considerar que se corresponden con la evaluación razonada de (o la discusión filosófica en torno a) un esquema genérico -que llamaremos meta-esquema abductivo-que autorizaría la utilización de una expresión del tipo "H explica D" (o " $\mathrm{H}$, de ahí que D") como la garantía de un argumento abductivo concreto " $\mathrm{D}$, por tanto H".

El llamado meta-esquema abductivo tendría la siguiente estructura y sería lo que los filósofos amigos de o contrarios a la abducción discutirían en sus textos:

\begin{tabular}{lc}
\cline { 2 - 2 } & H explica D \\
\cline { 2 - 2 } $\begin{array}{l}\text { Que una hipótesis explique unos } \\
\text { datos es una razón para inferirla } \\
\text { de dichos datos: }\end{array}$ & Por tanto \\
\cline { 2 - 2 } & $\begin{array}{l}\text { H se puede inferir de D } \\
\text { (o D puede sustentar H) }\end{array}$ \\
\hline
\end{tabular}

Habría diversos modos de defender o, en su caso, de atacar, cuestionar o restringir la aceptabilidad de este argumento genérico o, en terminología de Toulmin, warrant-establishing argument, es decir, argumento dirigido al establecimiento de una garantía tipo (Toulmin, 2003[1958]: 111). En lo que sigue, trataremos de catalogar y analizar algunos de los más reconocidos en la literatura filosófica, revisando sus características argumentativas, teniendo en cuenta que las discusiones sobre la justificación de la abducción se han visto animadas por su incidencia en el debate sobre el realismo científico (Diéguez, 1998; Iranzo, 2008), puesto que la abducción supone un modo de dar razones a favor de una hipótesis explicativa que podría incluir su operatividad efectiva y en muchos casos la referencia exitosa de las entidades, procesos y relaciones (muchos de ellos inobservables o al menos inobservados) que menciona.

Para comprender mejor en qué elementos del llamado meta-esquema abductivo inciden los diversos cuestionamientos, resulta útil contar con un diagrama ampliado en el que "H explica D" aparece como un acto más de dar razones con su propia garantía explicativa, utilizando la misma lógica representativa que anteriormente mencionábamos en el caso del modelo meta-explicativo de abducción. El diagrama ampliado o desplegado del meta-esquema abductivo sería el siguiente: 
Que una hipótesis explique unos datos es una razón para inferirla de dichos datos:

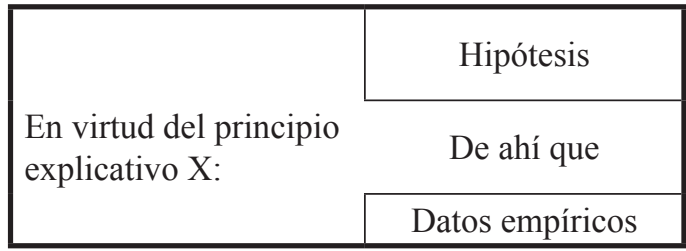

Por tanto

H se puede inferir de D

La discusión filosófica sobre la aceptabilidad de este argumento genérico -y generador, a su vez, de argumentos abductivos particulares- puede desembocar fácilmente, y es algo que sucede de manera típica, dada la metodología propia de la filosofía de tradición analítica, en la "exigencia" de alguna condición o restricción adicional.

\section{A. Restricción de campo (dialéctico-procedimental)}

Así, el meta-esquema podría aceptarse en ciertos contextos o prácticas argumentativas y no en otros (e.g. sí en la vida corriente y no en la práctica científica), lo que determinaría la admisión en los primeros, pero no en los segundos, de los argumentos abductivos. Y esto podría, a su vez, apoyarse en diversas razones:

1. Razones del mayor/menor rigor exigido a las pruebas y argumentos en dicho campo.

2. Razones de mayor/menor presencia de la necesidad o requisito institucional de alcanzar una conclusión.

Como es obvio, estos dos tipos de consideraciones actuarían, en las situaciones argumentativas particulares, en sentidos opuestos.

\section{B. Restricción de la pretensión}

El meta-esquema podría aceptarse sometiéndose a la exigencia de un ajuste en la pretensión de validez de la conclusión. Así, J. Woods (2016) considera que si bien la abducción no aporta razones suficientes para justificar la aserción de la hipótesis explicativa, sí justifica algo así como su pre-selección como candidata a ulteriores pruebas, lo que, en cierto modo, le estaría llevando a tratar la abducción como un argumento práctico en la línea que hemos 
señalado anteriormente. Su modo de defender la abducción, en este sentido mínimo, podría representarse como la aceptación de un meta-esquema abductivo modificado del siguiente modo:

\begin{tabular}{|l|c|}
\hline \multirow{2}{*}{$\begin{array}{l}\text { En virtud del principio } \\
\text { explicativo X: }\end{array}$} & Hipótesis \\
\cline { 2 - 2 } & Datos empíricos \\
\hline
\end{tabular}

Que una hipótesis explique unos datos es una razón para poner a prueba dicha hipótesis a la vista de tales Por tanto datos:

\section{Debemos poner prueba $\mathbf{H}$}

En un sentido muy similar (y como concreción experimental de las exigencias de dicha propuesta práctica) podrían interpretarse las conocidas precisiones de I. Hacking en Representar e intervenir (1996, [1983], 300-301) en torno a la debilidad de la abducción para sostener, por sí sola, un juicio realista sobre determinada entidad teórica.

Como es sabido, Hacking exige varios tipos o grados de prueba experimental (i.e. exige, finalmente, una conjunción de argumentos) que comportarían la detección y, finalmente, la manipulación de tales entidades para considerar justificada su aceptación como entidades reales (cf. Douven, 2002, 360-362). El modelo de justificación científica de una pretensión realista propuesto por Hacking, que iría más allá de la abducción pero que, en cierto modo la contendría, podría representarse (de manera bastante esquemática pero clarificadora) del siguiente modo ${ }^{8}$ :

\begin{tabular}{|c|c|c|c|c|c|}
\hline \multirow{3}{*}{$\begin{array}{l}\text { En virtud } \\
\text { del Principio } \\
\text { Explicativo } \mathrm{X} \text { : }\end{array}$} & $\begin{array}{l}\text { Hipótesis (incluye } \\
\text { el postulado de la } \\
\text { entidad "e") }\end{array}$ & \multirow[t]{3}{*}{ Además } & \multirow{3}{*}{$\begin{array}{c}\text { Hemos } \\
\text { detectado } \\
\text { "e" }\end{array}$} & \multirow[t]{3}{*}{ Además } & \multirow{3}{*}{$\begin{array}{c}\text { Hemos } \\
\text { manipulado } \\
\text { "e"e }\end{array}$} \\
\hline & De ahí que & & & & \\
\hline & Datos & & & & \\
\hline \multicolumn{6}{|c|}{ Por tanto } \\
\hline \multicolumn{6}{|c|}{$\begin{array}{l}\text { Hipótesis } \\
\text { tensión realista sobre "e") }\end{array}$} \\
\hline
\end{tabular}

\footnotetext{
${ }^{8}$ En este diagrama se utilizan algunas convenciones que van más allá de lo introducido en el presente trabajo, incluyendo la caracterización de estructuras argumentativas de combinación de argumentos coorientados, y que se pueden encontrar descritas con mayor detalle en Marraud (2013, 56-63).
} 


\section{Restricción sobre las características de la explicación invocada}

En otros casos, el meta-esquema abductivo se ha considerado aceptable como razonable solo en caso de que se complete con algún tipo de exigencia normativa que lo restrinja:

C.1. Sobre los "tipos de patrón explicativo" aceptables en un determinado campo, lo que sería muy propio de la actividad científica donde se discuten corrientemente patrones de explicación y principios explicativos aceptables e inaceptables en el sentido de "científicos" o "no científicos". A nuestros efectos, lo importante de este tipo de restricción está en ver cómo obliga a explicitar de un modo preciso la garantía explicativa que habilita la garantía argumentativa de las abducciones.

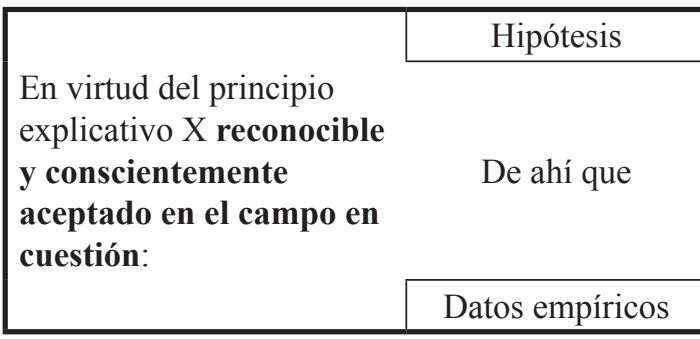

Que una hipótesis explique unos datos de este modo es una razón para inferirla de Por tanto dichos datos:

H se puede inferir de D

C.2. O (generalmente "además", i.e. pidiendo una conjunción de razones a su favor) sobre la calidad del cumplimiento del patrón explicativo en cuestión; ya sea en versión "cualitativa" ("Se trata de una buena explicación") o "comparativa" ("Se trata de una explicación mejor que las alternativas disponibles"). Como veremos más adelante (ver. E), el caso comparativo nos lleva directamente a un modelo en la línea de la llamada inferencia de la mejor explicación, $\mathrm{y}$, en nuestro caso, a considerar el interés de su versión argumentativa, es decir, el interés de tratar de comprender el tipo de discusión razonada que lleva al establecimiento de premisas del tipo "Se trata de una explicación mejor que las alternativas disponibles", más allá de su mera exigencia. 
Que una hipótesis explique unos datos de este modo es una razón para inferirla de dichos datos:

\begin{tabular}{|l|c|c|}
\hline \multicolumn{1}{l}{$\begin{array}{l}\text { En virtud } \\
\text { del principio } \\
\text { explicativo X } \\
\text { reconocible y } \\
\text { conscientemente } \\
\text { aceptado en } \\
\text { el campo en } \\
\text { cuestión: }\end{array}$} & De ahí que & $\begin{array}{c}\text { Se trata de } \\
\text { una buena } \\
\text { explicación / } \\
\text { Se trata de una } \\
\text { explicación } \\
\text { mejor que las } \\
\text { alternativas } \\
\text { disponibles }\end{array}$ \\
\hline
\end{tabular}

Por tanto

H se puede inferir de D

\section{Restricción epistémica (petición de respaldo)}

Ante las suspicacias que, en todo caso, sigue despertando la abducción como un modo de justificar hipótesis, algunos filósofos han aducido que aceptar el tipo de garantía que consideramos propia del meta-esquema abductivo presupone (en todo caso) la aceptación, a su vez, de algún principio epistémico adicional que apoye su validez. En terminología de Toulmin esto equivale a solicitar el respaldo de la garantía, es decir, equivale a un tipo de cuestionamiento que supone pedir razones adicionales a favor de su aceptabilidad. El respaldo actúa como la premisa de un argumento a favor de la garantía, creando la posibilidad de una estructura recursiva que representa la inagotable posibilidad de seguir argumentado y dando razones.

Como propuestas concretas para encarnar dicho respaldo podemos reconstruir la oposición entre las posturas de algunos filósofos relativamente favorables a la aceptación de la abducción, como P. Lipton (1991, 1993), y un oponente declarado tanto de la abducción como del realismo científico como B. van Fraassen (1989) (cf. Douven, 2002, 356-360). La estrategia de ambos es tratar de determinar el principio epistémico en el que se apoyaría la aceptabilidad de la garantía abductiva, pero sus propuestas encarnan visiones opuestas.

D.1. Lipton enuncia un principio epistémico más o menos discutible: "La mejor explicación (se entiende que disponible) es probablemente la explicación más plausible" ("The loveliest explanation is probably the likeliest explanation”): 


\begin{tabular}{|c|c|c|c|c|}
\hline & \multirow{3}{*}{$\begin{array}{l}\text { En virtud del } \\
\text { principio explicativo } \\
\text { X reconocible y } \\
\text { conscientemente } \\
\text { aceptado en el } \\
\text { campo en cuestión: }\end{array}$} & Hipótesis & \multirow{3}{*}{$\mathrm{Y}$} & \multirow{3}{*}{$\begin{array}{l}\text { Se trata de } \\
\text { una buena } \\
\text { explicación / } \\
\text { Se trata de una } \\
\text { explicación } \\
\text { mejor que las } \\
\text { alternativas } \\
\text { disponibles }\end{array}$} \\
\hline $\begin{array}{c}\text { La mejor explicación } \\
\text { disponible es } \\
\text { probablemente la } \\
\text { explicación más } \\
\text { plausible }\end{array}$ & & De ahí que & & \\
\hline Por tanto & & Datos empíricos & & \\
\hline \multirow[t]{2}{*}{$\begin{array}{l}\text { Que una hipótesis } \\
\text { explique unos datos } \\
\text { de este modo es una } \\
\text { razón para inferirla de } \\
\text { dichos datos: }\end{array}$} & \multicolumn{4}{|c|}{ Por tanto } \\
\hline & \multicolumn{4}{|c|}{ H se puede inferir de D } \\
\hline
\end{tabular}

D.2. Van Fraassen exige, en cambio, el cumplimiento de un principio extremadamente polémico: "Siempre o en la mayor parte de los casos la explicación verdadera se encuentra entre las disponibles", apoyado a su vez en una concepción casi "infalible" de la cognición humana: ya que "Los seres humanos contamos con una intuición privilegiada que nos garantiza un acceso a la verdad", que podemos representar de este modo:

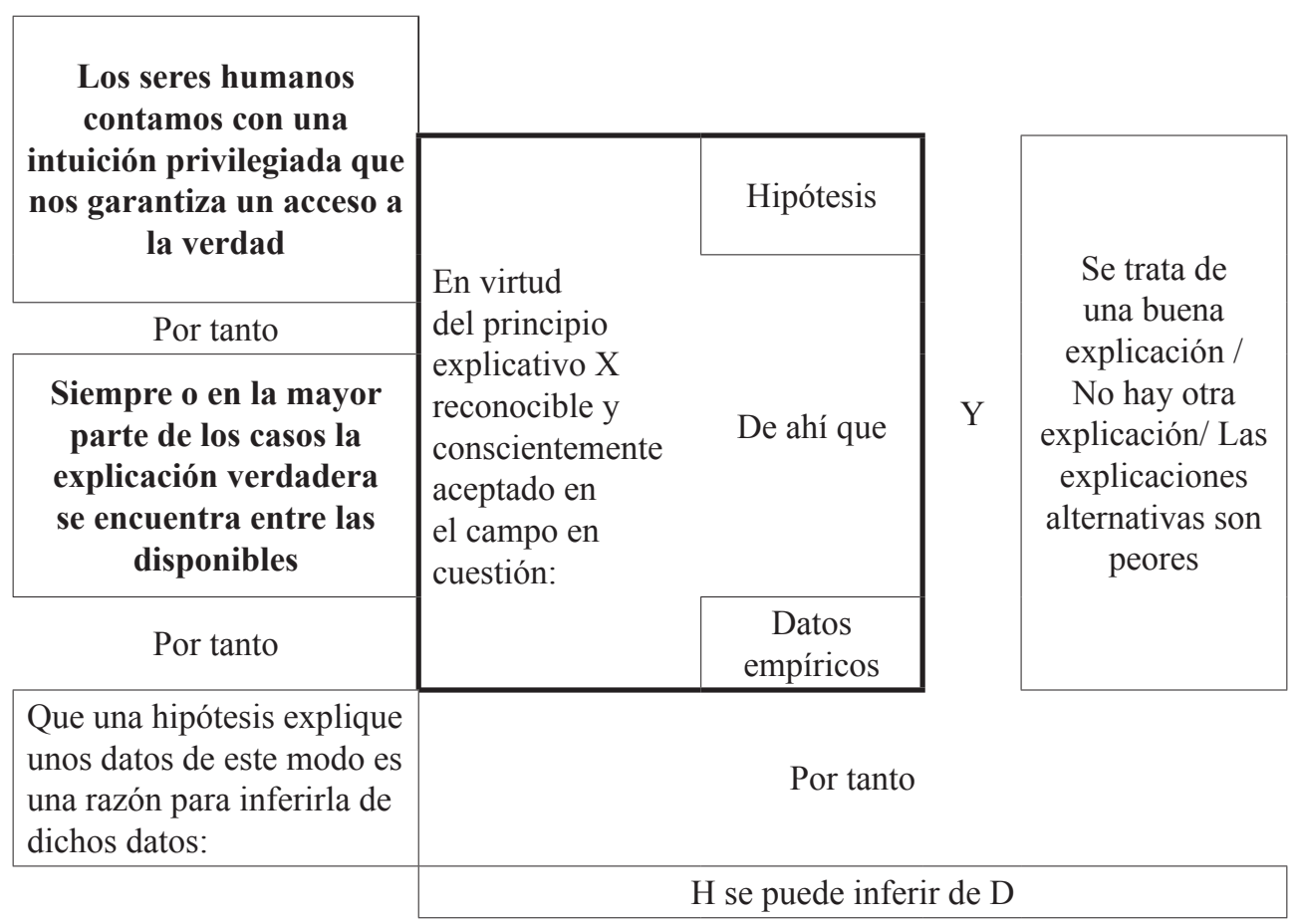


Lo que resulta, obviamente, difícil de aceptar y le lleva a considerar totalmente injustificada la abducción, aunque ello responde al punto de vista extremo y fundacionista de quienes, como decía Nagel "se niegan a otorgar la dignidad de conocimiento genuino a cuanto no está demostrado a partir de premisas prístinas y autoevidentes" (Cordero y Galparsoro, 2013, 9).

\section{E. Restricción ponderativa}

Finalmente, en una línea que completaría el tipo de discusión iniciado con las restricciones mencionadas en C, otros filósofos (e.g. I. Douven), considerarían que la aceptabilidad de un "argumento abductivo" quedaría enteramente pendiente de la aceptabilidad de la premisa valorativa introducida en C.2 ("Se trata de una explicación mejor que las alternativas disponibles"), lo que obligaría siempre a justificarla.

En este sentido, el reconocimiento de la garantía abductiva como concreción de un tipo de razón no sería "general" sino condicionado a tales procesos justificativos. La abducción en sí no sería razonable, sino como IME. Se exigiría la "ponderación justificada (argumentada) de las hipótesis explicativas disponibles", que, en mi opinión, puede presentar múltiples formas. Entre ellas que cabría mencionar, como particularmente interesante para la práctica justificativa de las ciencias empíricas, la consideración de la superioridad (o preferencia contextual) de algún patrón explicativo sobre otros, que podemos representar por medio de un argumento ponderativo (i.e. que comporta la evaluación comparativa de dos actos de dar razones, en este caso, explicativas). Podemos representar dicha ponderación del siguiente modo:

The loveliest

El principio explicativo $\mathrm{X}$ es superior al principio explicativo $\mathrm{Y}$ explanation is probably the likeliest explanation:

Por tanto

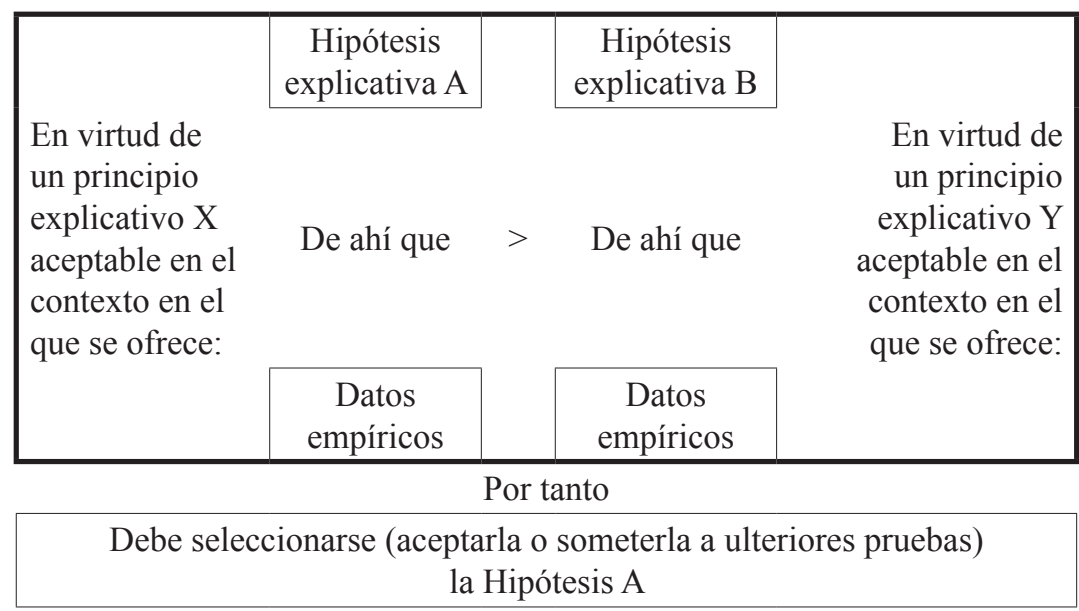




\section{Conclusiones}

En el presente trabajo he tratado de mostrar la pertinencia de un análisis argumentativo tanto de los modelos de justificación racional de las conclusiones científicas - centrándonos en particular, en un tipo de esquema central para dicha actividad como es el de la abducción (secciones 2 y 3) - como de los cuestionamientos filosóficos que el uso efectivo de tales modelos suscitan y las distintas soluciones propuestas ante tales demandas epistémicas (sección 4).

El resultado es, espero que al menos, clarificador de las diversas instancias y estrategias evaluativas que suscita la puesta en marcha de una práctica intrínsecamente normativa - tanto para los propios participantes como para el analista externo- cual sería la propuesta y defensa justificada de hipótesis teóricas; uno de los productos, si no el único sí el más conspicuo, de la práctica científica en tanto case-making profession (Woods, 2016, 143).

El análisis argumentativo parte, en todo caso, de la asunción del carácter conscientemente falibilista y antifundacionista de las prácticas de justificación racional de las ciencias empíricas (sección 1), en las que tanto los puntos de partida como las conclusiones se consideran, por un lado, solo provisionalmente establecidos pero, por otro, razonablemente justificados en el sentido de llegar a considerarse, gracias, precisamente, a su exigente discusión pública, "más allá de la duda razonable".

La labor filosófica que se asume en este contexto, es en primer lugar, la de poner de manifiesto la normatividad propia de la práctica probatoria o forense de los agentes científicos, encarnada en los modos de discusión y evaluación de sus propuestas justificativas que, atendiendo a los fines explicativos de su indagación, se encarna, de manera habitual, en estructuras racionales de carácter abductivo (uso de argumentos meta-explicativos). Es lo que he tratado de hacer en las secciones 2 y 3 .

En segundo lugar, también es posible atender a las particularidades argumentativas y pretensiones normativas de un nivel meta-discursivo, de carácter este ya filosófico, en el que se ponen en cuestión los propios principios (o garantías) invocados en dicha práctica evaluativa de primer nivel. Se trata, en este caso, de discutir cuáles son, si es que los hay, los límites reconocibles y justificables de aplicación de un modo específico de dar razones justificativas cual sería el modelo abductivo. Es lo que he tratado de hacer en la sección 4, reflejando diversas posturas más o menos sofisticadas en torno a la justificación epistémica de la abducción.

Sin que se abracen aquí las pretensiones o motivaciones fundacionistas que pueden adivinarse detrás de algunos de estos intentos justificacionistas, 
comprender y clarificar el modo en el que se discuten y se contraponen contribuye, a su vez, a la caracterización argumentativa de la propia práctica filosófica.

\section{Agradecimientos}

Agradezco a Obdulia Torres y a Ana Cuevas la organización en la Universidad de Salamanca, de las Jornadas Novatores 2018 "Enfoques contemporáneos del realismo científico", que dieron lugar a este trabajo y agradezco asimismo los comentarios y sugerencias de los asistentes a la sesión en la que se presentó y que me han ayudado a terminar de pulirlo y mejorarlo. La investigación que lo ha hecho posible se ha beneficiado, por otra parte, de la financiación del Proyecto FFI2014-53164-P del MINECO, "La construcción de agentes argumentativos en las prácticas del discurso público”.

\section{Referencias bibliográficas}

Álvarez, María (2010). Kinds of Reason. An Essay in the Philosophy of Action. Oxford: Oxford University Press.

Álvarez, María (2016). Reasons for Action: Justification, Motivation, Explanation. En Edward N. Zalta (Ed.) The Stanford Encyclopedia of Philosophy (Spring 2016 Edition), URL = https://plato.stanford.edu/archives/spr2016/entries/reasons-just-vs-expl/

Bermejo Luque, Lilian (2016). Modelo de Toulmin / Garantía / Respaldo / Reserva / Calificador. En Luis Vega y Paula Olmos (Eds.), Compendio de lógica, argumentación y retórica (pp. 408-410). Madrid: Trotta.

Cordero, Alberto (1997 [1991]). Las ideas evolucionistas y el naturalismo contemporáneo. En Sergio Martínez y León Olivé (Eds.), Epistemología evolucionista (pp. 185-219). México: Paidós.

Cordero, Alberto y Galparsoro, José Ignacio (eds.) (2013). Reflections on Naturalism. Boston: Sense Pub.

Diéguez Lucena, Antonio (1998). Realismo científico. Una introducción al debate actual en Filosofía de la Ciencia. Málaga: Universidad de Málaga.

Douven, Igor (2002). Testing Inference to the Best Explanation. Synthese, $130,355-377$.

Fraassen, Bas van (1989). Laws and Symmetry. Oxford: Clarendon Press.

Hacking, Ian (1996 [1983]). Representar e intervenir, trad. de Sergio Martínez. México: Paidós. 
Harman, Gilbert (1965). The Inference to the Best Explanation. Philosophical Review, 74, 88-95.

Iranzo, Valeriano (2007). Abduction and Inference to the Best Explanation. Theoria, 60, 339-346.

Iranzo, Valeriano (2008). El dilema del realismo experimental, Episteme NS 28(1), 59-88.

Lipton, Peter (1991). Inference to the Best Explanation. Londres: Routledge.

Lipton, Peter (1993). Is the best good enough? Proceedings of the Aristotelian Society, 93, 89-104

Marraud, Hubert (2013). ¿Es lógic@? Análisis y evaluación de argumentos. Madrid: Cátedra.

Marraud, Hubert (2017). Guia de Campo de Esquemas Argumentativos. Publicado on-line: https://www.academia.edu/

Olmos, Paula (2018). Narrative Reasons in Scientific Argument. En Steve Oswald y Didier Maillat (Eds.) Argumentation and Inference: Proceedings of the 2nd European Conference on Argumentation, Fribourg 2017, Vol. I (pp. 487-506). London: College Publications.

Olmos, Paula (en prensa). Un enfoque argumentativo sobre la abducción y sobre la ponderación de hipótesis explicativas. Theoria.

Pollock, John L. (1987). Defeasible Reasoning, Cognitive Science, 11, 481518.

Toulmin, Stephen E. (2003 [1958]). The uses of argument. Cambridge: Cambridge University Press.

Toulmin, Stephen E. (1961). Foresight and Understanding: An Enquiry into the Aims of Science. Bloomington: Indiana University Press.

Walton, Douglas, Reed, Chris y Macagno, Fabrizio (2008). Argumentation Schemes. Cambridge: Cambridge University Press.

Woods, John (2016). "Reorienting the Logic of Abduction". En L. Magnani y T. Bertolotti, eds., Springer Handbook of Model-Based Science. Dordrecht: Springer. 